\title{
Size-Dependent Self-Organization of Colloidal Particles on Chemically Patterned Surfaces
}

\author{
Ismail Karakurt, ${ }^{* \dagger}$ Paul Leiderer, ${ }^{\dagger}$ and Johannes Boneberg ${ }^{\ddagger}$ \\ Department of Physics, University of Konstanz, 78467, Konstanz, Germany, and Department of Physics, \\ Isik University, Kumbaba Mevkii, 34980 Sile, Istanbul, Turkey
}

Received August 12, 2005. In Final Form: January 31, 2006

\begin{abstract}
A study of the self-organization of colloidal particles during the evaporation of particle solutions on chemically patterned surfaces is presented. On a surface with hydrophilic and hydrophobic regions, colloidal particles form compact structures on the hydrophilic sites. When a colloidal solution containing a mixture of particles with a variation in size is used, the number density of each type of particle deposited on the hydrophilic islands after evaporation decreases with increasing particle size. This makes it possible to produce a concentration gradient of the particles on islands of different sizes. It is shown that this technique could allow for particle separation.
\end{abstract}

\section{Introduction}

Self-organization of colloidal particles is interesting and has been studied for the purposes of many applications including nanotechnology, ${ }^{1-3}$ photonics, ${ }^{4,5}$ and biotechnology. When a colloidal suspension is dispersed onto a clean surface, colloidal particles form compact structures on the surface as the solvent evaporates. Ordered monolayers may form because of capillary forces between the particles. The growth of monolayers is promoted if the contact angle is small so that only a monolayer of colloidal particles can be accommodated within the evaporating front of the liquid (i.e., the wetting of the substrate by the solvent is an important factor). Self-assembled compact structures or monolayers can also be produced on chemically patterned surfaces. In this case, patterning means creating either a contrast in terms of hydrophilicity $4,7,8$ or alternately charged regions ${ }^{9}$ on a surface. The hydrophilicity of a surface can be tailored by employing microcontact printing using $n$-alkyl molecules. To our knowledge, the size-dependent self-organization of colloidal particles has not been investigated. In this letter, we present a study of the self-organization of a mixture of particles with different diameters on chemically patterned Au surfaces. The number of particles deposited on the hydrophilic sites decreases with increasing particle diameter. For a given mixture, the deposition of the larger particles can be prevented by reducing the size of the printed features. We give a qualitative explanation of the exclusion of the larger particles from the smaller hydrophilic sites.

\section{Experiments and Results}

In Figure 1, we show an SEM image of $250 \mathrm{~nm}$ PS particles deposited on periodic $8 \times 8 \mu \mathrm{m}$ hydrophilic islands created using $n$-alkyl thiols on an Au surface. The stamp, which was used in $\mu \mathrm{CP}$

* Corresponding author. E-mail: ikarakurt@isikun.edu.tr.

$\dagger$ Isik University.

¥ University of Konstanz.

(1) Boal, A. K.; Ilhan, F.; DeRouchey, J. E.; Thurn-Albrecht, T.; Russell, T. P.; Rotello, V. M. Nature 2001, 404, 746.

(2) Burmeister, F.; Badowsky, W.; Braun, T.; Wieprich, S.; Boneberg, J.; Leiderer, P. Appl. Surf. Sci. 1999, 461, 144-145.

(3) Boneberg, J.; Burmeister, F.; Schaefle, C.; Leiderer, P.; Reim, D.; Fery A.; Herminghaus, S. Langmuir 1997, 13, 7080.

(4) Himmelhaus, M.; Takei, H. Phys. Chem. Chem. Phys. 2002, 4, 496.

(5) Fujimura, T.; Itoh, T.; Imada, A.; Shimada, R.; Koda, T.; Chiba, N.; Muramatsu, H.; Miyazaki, H.; Ohtaka, K. J. Lumin. 2000, 87-89, 954.

(6) Charles, M. H.; Charreyre, M. T.; Delair, T.; Elaissari, A.; Pichot, C. STP Pharma Sci. 2001, 11, 251.

(7) Fan, F.; Stebe, K. J. Langmuir 2004, 20, 3062.

(8) Schaefle, C.; Leiderer, P.; Bechinger, C. Europhys. Lett. 2003, 63, 394.

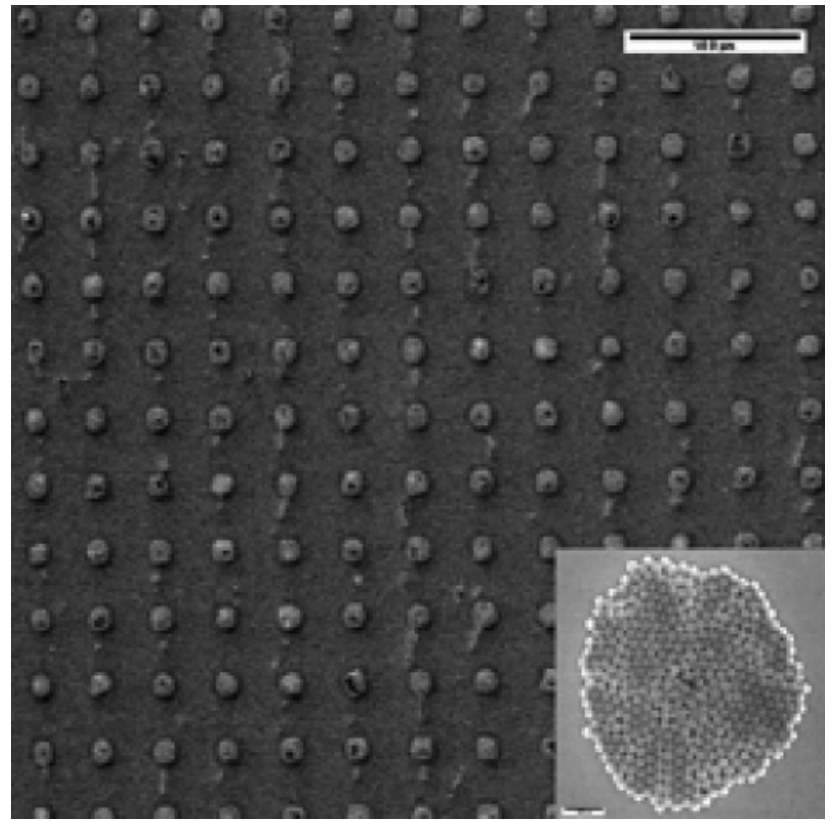

Figure 1. SEM picture of $250 \mathrm{~nm}$ PS spheres deposited on a microcontact-printed Au substrate. The bar is $50 \mu \mathrm{m}$.

(microcontact printing), was cast from a master prepared by photolithography. About $50 \mu \mathrm{L}$ of a $0.001 \%$ solution of polystyrene (PS) particles was left on the printed substrate. The drop evaporated in about $3 \mathrm{~h}$, leaving compact structures of PS particles on the hydrophilic sites. Taking into account the spread of the drop on the stamped area of the surface, this evaporation rate gives a contact line speed of $\sim 1 \mu \mathrm{m} / \mathrm{s}$. The image shows that whereas most of the structures are formed as monolayers of PS particles some have voids or isolated particles on top as a second layer. With the proper choice of particle concentration, periodic structures consisting of monolayers of particles can be obtained as shown in the inset of the Figure. Islands form at the center of hydrophilic sites and mimic the overall shape of the printed regions. The thickness of the Au on the glass substrates used in this study was $75 \mathrm{~nm}$. To improve the adhesion of $\mathrm{Au}$ onto the substrates, a layer of $\sim 2 \mathrm{~nm} \mathrm{Cr}$ was deposited onto the glass slides before the evaporation of $\mathrm{Au}$.

When a colloidal solution of $800 \mathrm{~nm}$ particles, instead of $250 \mathrm{~nm}$ particles, was placed on a surface with patterns of the same size as in Figure 1, the number of particles deposited on each site was

(9) Chen, K. M.; Jiang, Xueping, Kimerling, L. C.; Hammond, P. T. Langmuir 2000, 16, 7825 . 


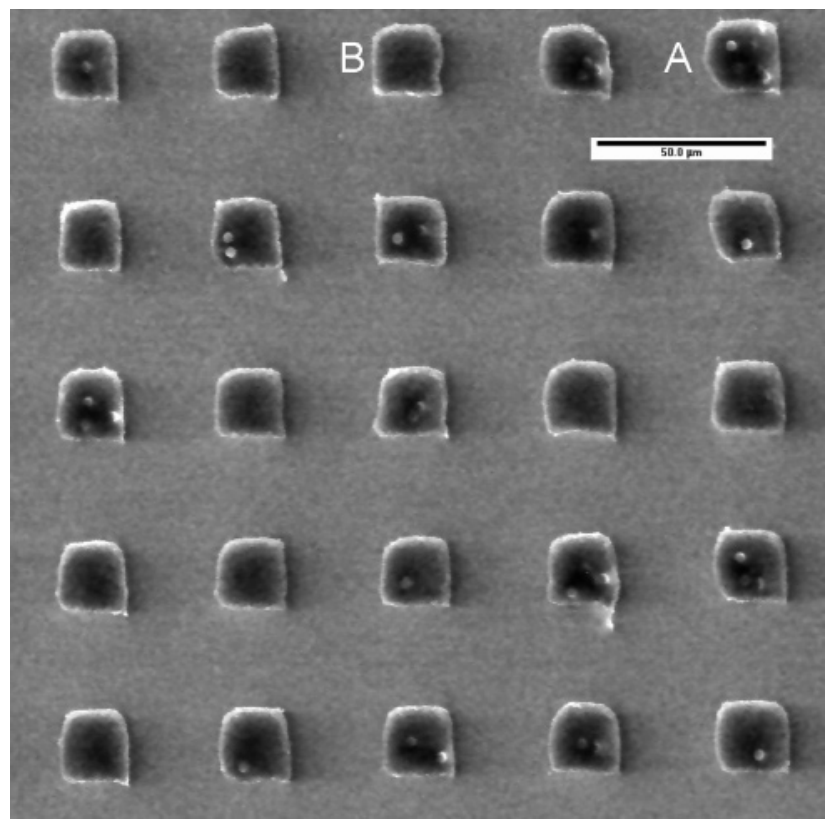

Figure 2. SEM picture of a mixture of PS spheres deposited on a microcontact-printed Au substrate. The mixture contains $3 \mu \mathrm{m}, 800$ $\mathrm{nm}$, and $250 \mathrm{~nm}$ particles. Hydrophilic regions are $22 \times 22 \mu \mathrm{m}$. The bar is $50 \mu \mathrm{m}$.

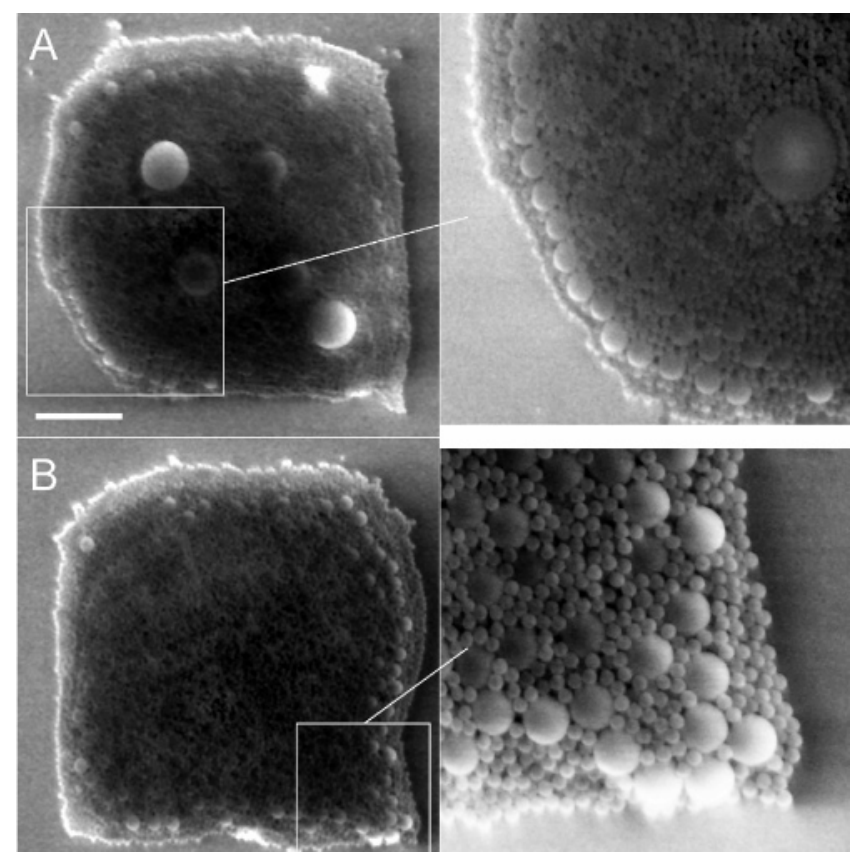

Figure 3. Blown-up images of islands A and B in Figure 2. The bar is $5 \mu \mathrm{m}$.

dramatically reduced. This led us to investigate the self-organization of a mixture of particles with different diameters. As a colloidal solution, we prepared a mixture of $3 \mu \mathrm{m}(0.00067 \mathrm{vol} \%), 800 \mathrm{~nm}$ (0.0005 vol \%), and $250 \mathrm{~nm}(0.00035 \mathrm{vol} \%)$ PS particles. Approximately $50 \mu \mathrm{l}$ of the solution was left to evaporate on microcontact printed substrates with periodic $22 \times 22 \mu$ m hydrophilic islands. The choice of the concentration of particles in the solution is important. The concentrations above were found to be optimal in terms of achieving deposition on only the hydrophilic sites on the printed surfaces used in our experiments. When the concentrations are too high, some particles that are randomly scattered over the hydrophobic regions between the hydrophilic sites are also observed. For concentrations that are too small, after the particle arrangement is completed, the hydrophilic sites contain very few particles, as one would expect.

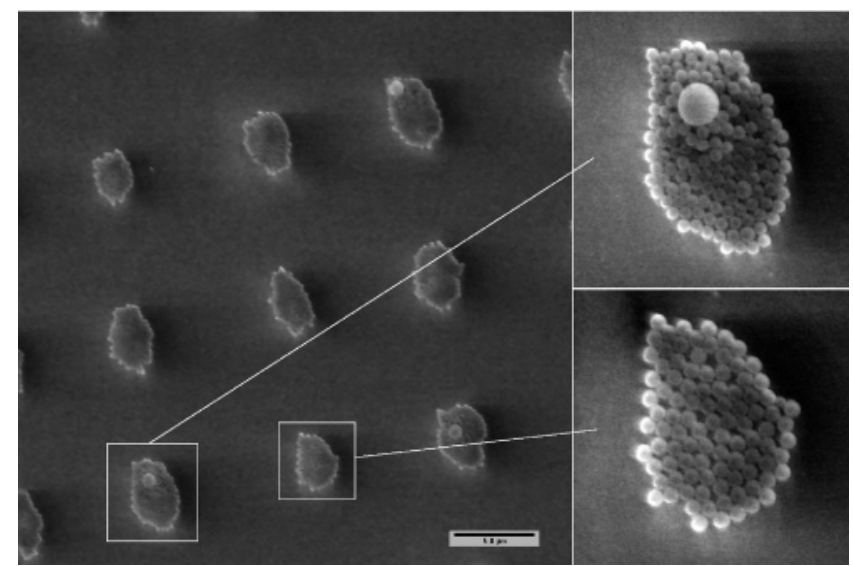

Figure 4. SEM picture of a mixture of PS spheres deposited on a microcontact-printed Au substrate. The mixture contains $3 \mu \mathrm{m}, 800$ $\mathrm{nm}$, and $250 \mathrm{~nm}$ particles. Hydrophilic regions are $7 \times 4.5 \mu \mathrm{m}$. The bar is $5 \mu \mathrm{m}$.
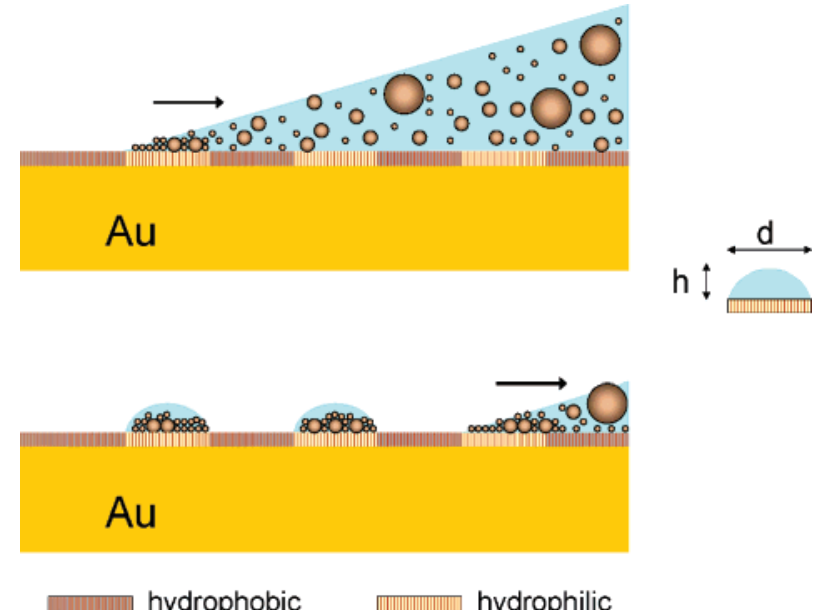

Figure 5. Schematic of the self-organization of a mixture of colloidal particles on a chemically patterned surface. The drawing is not to scale.

In Figure 2, we show an SEM image of compact structures formed upon evaporation of the colloidal mixture. The largest particles protruding in some of the islands in the Figure are the $3 \mu \mathrm{m}$ particles. Islands A and B in Figure 2 are shown in Figure 3 for a better view of the compact structures. The blown-up images of sections of both islands are shown on the right side of the Figure. In island A, five $3 \mu \mathrm{m}$ particles are seen to protrude at the surface and are surrounded by smaller particles. We see $800 \mathrm{~nm}$ particles forming compact structures in the central parts of the islands. Monolayers of these particles are surrounded by the $250 \mathrm{~nm}$ particles, which mostly form multiple layers. The smallest particles fill the gaps between larger particles and even bury some of them. The blown-up image of island A shows $250 \mathrm{~nm}$ PS spheres forming a ring around the $3 \mu \mathrm{m}$ particle. The number of particles in each island decreases with increasing particle size.

To see how the particle arrangement is affected by the size of hydrophilic sites, we used the same colloidal mixture on another substrate with smaller hydrophilic periodic islands. Because of the features on the master from which the stamp for microcontact printing was cast, the hydrophilic sites had an elliptical geometry with dimensions of $7 \times 4.5 \mu \mathrm{m}$.

In Figure 4, we show an SEM image of the particles deposited on these smaller hydrophilic islands. It is seen, in this case, that the islands consist of mostly $250 \mathrm{~nm}$ particles. A few of the islands also contain single $800 \mathrm{~nm}$ particles whereas the $3 \mu \mathrm{m}$ particles are not deposited at all. The enlarged images on the right show that the islands with only $250 \mathrm{~nm}$ particles are largely in the form of 
Table 1. Comparison of the Average Numbers of Particles of Each Type That Were Deposited on the Two Types of Hydrophilic Sites

\begin{tabular}{|c|c|c|c|c|c|c|}
\hline $\begin{array}{c}\text { particle } \\
\text { size }(n m)\end{array}$ & $\begin{array}{c}\text { number of } \\
\text { particles on } \\
22 \times 22 \mu \mathrm{m} \\
\text { squares }\end{array}$ & $\begin{array}{l}\text { normalized } \\
\text { values on } \\
22 \times 22 \mu \mathrm{m} \\
\text { squares }\end{array}$ & $\begin{array}{c}\text { number of } \\
\text { particles on } \\
7 \times 4.5 \mu \mathrm{m} \\
\text { ellipses }\end{array}$ & $\begin{array}{c}\text { normalized } \\
\text { values on } \\
7 \times 4.5 \mu \mathrm{m} \\
\text { ellipses }\end{array}$ & $\begin{array}{c}\text { original } \\
\text { concentration } \\
\text { in solution } \\
\left(\text { particles } / \mathrm{cm}^{3}\right)\end{array}$ & $\begin{array}{c}\text { normalized } \\
\text { values in } \\
\text { solution }\end{array}$ \\
\hline 250 & 13700 & 0.963 & 109 & 0.999 & $4280 \times 10^{7}$ & 0.957 \\
\hline 800 & 520 & 0.037 & 0.12 & $1.1 \times 10^{-3}$ & $187 \times 10^{7}$ & 0.0418 \\
\hline 3000 & 1.3 & $0.914 \times 10^{-4}$ & 0 & 0 & $4.75 \times 10^{7}$ & $1.06 \times 10^{-3}$ \\
\hline
\end{tabular}

monolayers. Those with an $800 \mathrm{~nm}$ particle show also a ring-shaped second layer formed by the smaller particles around the larger particle.

As statistical information we give the average numbers of particles of each type that were deposited on hydrophilic sites in Table 1 . The data for the deposition on $22 \times 22 \mu \mathrm{m}$ square sites are obtained by averaging the counts over 29 sites, whereas for $7 \times 4.5 \mu$ m elliptical sites the averaging was over 35 sites. Reducing the size of the hydrophilic sites results in a big difference in terms of both the total number and the type of deposited particles. A comparison with the data for the $22 \times 22 \mu \mathrm{m}$ square sites shows that the $3 \mu \mathrm{m}$ particles are not deposited on the elliptical hydrophilic sites of $7 \times 4.5 \mu \mathrm{m}$. The average number of $800 \mathrm{~nm}$ particles deposited on a given site is much less than 1, and the islands are formed nearly only by the $250 \mathrm{~nm}$ particles.

\section{Discussion}

A schematic view of the self-organization of a mixture of colloidal particles by evaporation is given in Figure 5. When a drop of a mixture is put on a surface with hydrophilic and hydrophobic regions, the receding front ${ }^{7,10}$ of the evaporating drop gets pinned on the hydrophilic sites. We observed under a microscope that, as the evaporation continues, the mother drop detaches from these sites, where it leaves small droplets containing colloidal particles, and jumps onto the next hydrophilic site. These small droplets then evaporate and form compact periodic structures on the substrate. The compact structures form at the central part of the islands because evaporation forces the droplets to recede from the perimeter of the sites.

The receding contact angle of the mother drop containing colloidal particles depends on the geometry of the chemically patterned surface. ${ }^{7}$ Which particles are deposited on the hydrophilic regions depends on both the size of the particles and the cap height of the small droplets, which is affected by the receding contact angle. ${ }^{7}$ Using optical methods, we measure the receding contact angles to be $48-52^{\circ}$ on both of the patterned surfaces used. These values are consistent with the previous measurements on similar patterned surfaces. ${ }^{7}$ Assuming the caps to be spherical, the above contact angles yield a cap height of $\sim 5.1 \mu \mathrm{m}$ for the droplets on $22 \times 22 \mu \mathrm{m}$ squares and a cap height of $\sim 1.1 \mu \mathrm{m}$ for the droplets on $7 \times 4.5 \mu \mathrm{m}$ ellipses.

Geometrically, it can be seen that if $\delta \approx h$, where $\delta$ denotes the particles' diameter, only a few of the particles may be accommodated in the droplets. We see experimentally that these particles are scattered randomly within the islands. The number of particles accommodated in the droplets is high when $\delta \ll h$. Therefore, the concentration of the smallest particles on the

(10) Deegan, R. D. Phys. Rev. E 2000, 61, 475. hydrophilic sites is always higher than that of the larger particles. For a particle to be excluded from the small droplets, its diameter, $\delta$, must be larger than the cap height, $h$.

The exclusion of the larger particles from the droplets on hydrophilic sites can be explained as follows. As the mother drop containing the colloidal particles evaporates, particles move toward the perimeter where the evaporation rate is high. Some of these particles get trapped at the air/water interface of the receding front (i.e., at the surface energy well ${ }^{11}$, which is much larger than $k_{\mathrm{B}} T$ ). The small particles (i.e., those for which $\delta \ll$ $h$ ) can move into the droplets forming on hydrophilic sites and therefore stay behind the breaking points of the detaching mother drop. As a result, these particles would be accommodated over the hydrophilic sites and within the droplets. For $\delta>h$, the particles that reach the perimeter get trapped at the interface before they can get past the breaking points and move into the forming droplets. These particles would then be taken away by the receding front of the mother drop.

\section{Conclusions}

In summary, we studied the self-organization of colloidal particles on surfaces that were chemically patterned using $n$-alkyl thioles. When a suspension of a mixture of colloidal particles is dispersed on a patterned surface, particles form compact structures on the hydrophilic sites. Whether a particle is deposited on a hydrophilic island depends on its size, the size of the island, and the receding contact angle of the evaporating liquid. The concentration of particles in the suspension is another important parameter because for concentrations that are too high the particles tend to be deposited randomly even outside the hydrophilic islands on the substrate. A gradient in the number density of particles that are deposited on the hydrophilic sites can be produced and modified simply by an adjustment in the size and periodicity of the printed features. Self-assembly by evaporation on chemically patterend surfaces offers a simple and inexpensive method that could be used to separate particles in a colloidal mixture. This may have extensive biological applications because the ability to separate individual cells and molecules in a mixture would be very useful in biological spectroscopy.

Acknowledgment. This work was supported by the Kompetentznetz Funktionelle Nanostrukturen Baden-Württemberg and the Deutsche Forschungsgemeinschaft (SFB 513).

\section{LA052205X}

(11) Pieranski, P. Phys. Rev. Lett. 1980, 45, 569. 